Herz und Lunge o.k.

\title{
Woher kommt die Atemnot?
}

\author{
Bei Belastungsdyspnoe zunächst an eine kardiale oder pulmonale Erkrankung \\ zu denken, liegt auf der Hand. Was aber, wenn Herz und Lunge offensichtlich \\ völlig in Ordnung sind? Im Falle einer 47-jährigen Patienten sorgte ein ganz \\ anderes Organ für Atemnot.
}

- Die Patientin stellte sich wegen zunehmender Dyspnoe, v.a. bei körperlicher Belastung, in der Hausarztpraxis vor. Die klinische Untersuchung ergab abgesehen von einer kleinen Struma keinen organpathologischen Befund, insbesondere keine Hinweise für eine bronchiale Obstruktion oder eine Herzinsuffizienz. Das EKG fiel unauffällig aus.

\section{Untersuchungen auf Herz und Lunge} Zur weiteren Abklärung wurde die Patientin zunächst einem Kardiologen vorgestellt. Die Farbdopplerechokardiografie ergab keinerlei Anhalt für ein hämodynamisch wirksames Vitium cordis oder eine Herzinsuffizienz. Bei der Ergometrie klagte die Patientin über zunehmende Luftnot. Hinweise auf eine hämodynamisch wirksame koronare Herzerkrankung gab es jedoch nicht.

Anschließend erfolgte eine pulmonologische Untersuchung. Bei der Lungenfunktionsuntersuchung fand sich eine leichte obstruktive Ventilationsstörung. Eine probatorische Inhalationstherapie mit einer Kombination aus Steroid mit
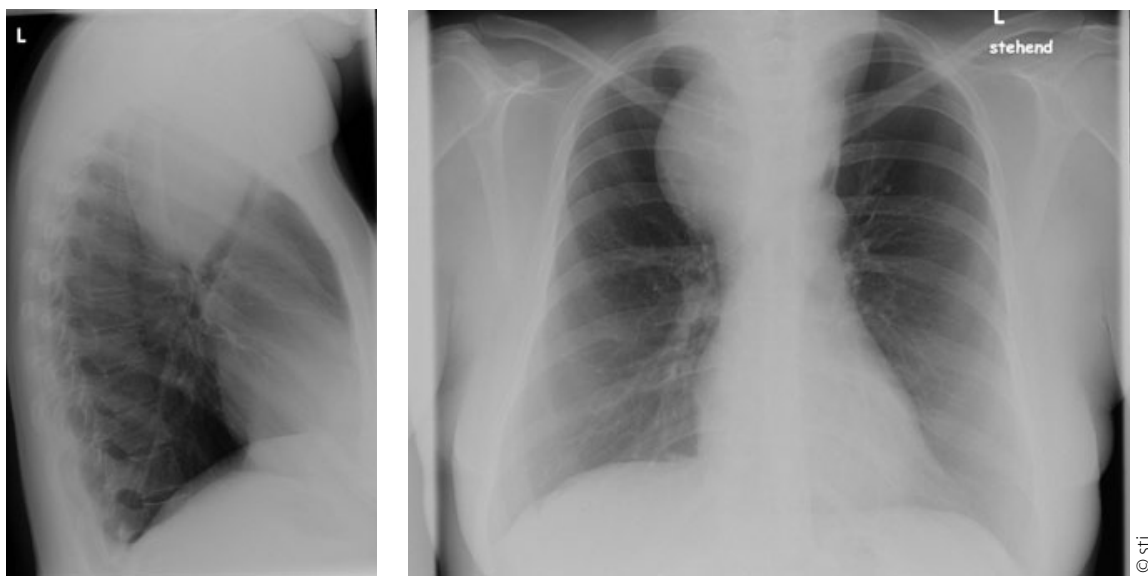

Röntgen-Thorax: große mediastinale Raumforderung mit Einengung der Trachea. einem Beta-1-Agonisten führte nicht $\mathrm{zu}$ einer Besserung der Symptomatik.

\section{Mediastinale Raumforderung im Röntgenbild}

In der nun veranlassten Röntgen-Thorax-Aufnahme fand sich eine ausgedehnte mediastinale Raumforderung im oberen Mediastinum. Die glatte Begrenzung sprach gegen einen malignen Prozess. Differenzialdiagnostisch wurde neben einem Thymom auch ein Lymphom diskutiert. Die anschließend durchgeführte computertomografische Untersuchung mit Kontrastmittel zeigte in der Raumforderung einen gleich hohen Dichteanstieg wie in der Schilddrüse. Somit handelte es sich um eine intrathorakale Struma, was szintigrafisch bestätigt werden konnte.

Die Struma führte zu einer Verdrängung der Trachea nach links und zu einer Kompression mit einem Restdurchmesser von $6 \mathrm{~mm}$. Hinweise für eine Schilddrüsenfunktionsstörung ergaben sich nicht. Es wurde die Indikation für die Strumektomie gestellt.

\section{Schilddrüse auf Abwegen}

Große intrathorakale bzw. retrosternal reichende Strumen sind selten. Sie können sich auch paratracheal oder retrotracheal ausdehnen. Meist besteht eine Parenchymbrücke zur Schilddrüse. Selten handelt es sich um eine aberrierende Struma im Sinne von dystopem Schilddrüsengewebe, das über eine eigene intrathorakale Gefäßversorgung verfügt, aber nicht über eine Parenchymbrücke mit der Schilddrüse in Verbindung steht.

Typischerweise zeigt das konventionelle Röntgenbild eine glatt begrenzte mediastinale Raumforderung des vorderen und mittleren Mediastinums, wobei auch Verkalkungen möglich sind. Das para- und retrotracheale Wachstum kann zu einer Trachealverlagerung bzw. einer Trachealkompression führen.

Differenzialdiagnostisch müssen bei einer umschriebenen Mediastinalverbreiterung auch Lymphome, bronchogene Zysten, Ösophaguserkrankungen (Ösophagusdivertikel, Ösophagustumor) oder Aneurysmen des Aortenbogens diskutiert werden. Auch eine Hiatushernie bzw. ein Upside-down-Magen kann ein solches Bild hervorrufen.

Mit der Schilddrüsenszintigrafie gelingt die eindeutige Abgrenzung der Struma von anderen Raumforderungen. Im CT zeigt die Raumforderung nach Kontrastmittelgabe einen gleich hohen Dichteanstieg wie die Schilddrüse.

Dr. med. Peter Stiefelhagen .

\section{Fabula docet}

Bei einer Belastungsdyspnoe sollte neben einer pulmonalen bzw. kardialen Erkrankung immer an eine intrathorakale Raumforderung gedacht werden. Bei den mediastinalen Raumforderungen muss neben einem Lymphom, einem Aneurysma und einem Upside-downMagen auch eine retrosternale Struma diskutiert werden. 